\title{
MicroRNA-125a-mediated regulation of the mevalonate signaling pathway contributes to high glucose-induced proliferation and migration of vascular smooth muscle cells
}

\author{
DAN YE ${ }^{1 *}$, GUO-HUA LOU ${ }^{2 *}$, AI-CHUN LI ${ }^{2}$, FENG-QIN DONG ${ }^{1}$, GUO-PING CHEN ${ }^{1}$, \\ WEI-WEI XU ${ }^{1}$, YAN-NING LIU ${ }^{2}$ and SHEN-JIANG HU ${ }^{3}$ \\ ${ }^{1}$ Department of Endocrinology and Metabolism; ${ }^{2}$ State Key Laboratory for Diagnosis and Treatment of Infectious Diseases, \\ National Clinical Research Center for Infectious Diseases, Collaborative Innovation Center for Diagnosis and Treatment of \\ Infectious Diseases; ${ }^{3}$ Institute of Cardiology, The First Affiliated Hospital of School of Medicine, \\ Zhejiang University, Hangzhou, Zhejiang 310003, P.R. China
}

Received May 27, 2019; Accepted March 11, 2020

DOI: $10.3892 / \mathrm{mmr} .2020 .11077$

\begin{abstract}
Hyperglycemia contributes to the excessive proliferation and migration of vascular smooth muscle cells (VSMC), which are closely associated with atherosclerosis. MicroRNAs (miRNAs/miRs) constitute a novel class of gene regulators, which have important roles in various pathological conditions. The aim of the present study was to identify miRNAs involved in the high glucose (HG)-induced VSMC phenotype switch, and to investigate the underlying mechanism. miRNA sequencing and reverse transcription-quantitative PCR results indicated that inhibition of miR-125a expression increased the migration and proliferation of VSMCs following HG exposure, whereas the overexpression of miR-125a abrogated this effect. Furthermore, dual-luciferase reporter assay results identified that 3-hydroxy-3-methyglutaryl-coA reductase (HMGCR), one of the key enzymes in the mevalonate signaling pathway, is a target of miR-125a. Moreover, HMGCR knockdown, similarly to miR-125a overexpression, suppressed HG-induced
\end{abstract}

Correspondence to: Dr Shen-Jiang Hu, Institute of Cardiology, The First Affiliated Hospital of School of Medicine, Zhejiang University, 79 Qingchun Road 6A-17, Hangzhou, Zhejiang 310003, P.R. China

E-mail: hzhz185519933@zju.edu.cn

Dr Yan-Ning Liu, State Key Laboratory for Diagnosis and Treatment of Infectious Diseases, National Clinical Research Center for Infectious Diseases, Collaborative Innovation Center for Diagnosis and Treatment of Infectious Diseases, The First Affiliated Hospital of School of Medicine, Zhejiang University, 79 Qingchun Road 6A-17, Hangzhou, Zhejiang 310003, P.R. China

E-mail: liuyanning@zju.edu.cn

*Contributed equally

Key words: vascular smooth muscle cells, microRNA-125a, 3-hydroxy-3-methyglutaryl-coA reductase, mevalonate pathway, high glucose
VSMC proliferation and migration. These results were consistent with those from the miRNA target prediction programs. Using a rat model of streptozotocin-induced diabetes mellitus, it was demonstrated that miR-125a expression was gradually downregulated, and that the expressions of key enzymes in the mevalonate signaling pathway in the aortic media were dysregulated after several weeks. In addition, it was found that HG-induced excessive activation of the mevalonate signaling pathway in VSMCs was suppressed following transfection with a miR-125a mimic. Therefore, the present results suggest that decreased miR-125a expression contributed to HG-induced VSMC proliferation and migration via the upregulation of HMGCR expression. Thus, miR-125a-mediated regulation of the mevalonate signaling pathway may be associated with atherosclerosis.

\section{Introduction}

Diabetes mellitus (DM) belongs to a group of chronic metabolic diseases characterized by chronic hyperglycemia and disturbance of fat and protein metabolism, which occur as a result of insulin deficiency and/or resistance, thus leading to elevated blood glucose levels and abnormal fat and protein metabolism $(1,2)$. Long-term hyperglycemia can result in microvascular and macrovascular damage, particularly cardiovascular disease (CVD) complications, which increase the morbidity and mortality rates of patients with diabetes (3). Furthermore, atherosclerosis is a major risk factor for DM-related CVD (4), and it is accelerated in type 1 and type 2 DM (5). Previous studies have shown that high glucose (HG)-induced migration and proliferation of vascular smooth muscle cells (VSMCs) are associated with the progression of atherosclerosis in DM $(4,6)$.

MicroRNAs (miRNAs/miRs) are endogenous non-coding RNAs, 20-24 nucleotides in length, which have a variety of important regulatory roles in cells (7). miRNAs inhibit translation or degrade mRNA by interacting with the 3'-untranslated region $\left(3^{\prime}-\mathrm{UTR}\right)$ of a target mRNA $(7,8)$. Moreover, via this interaction miRNAs modulate several pathological and 
physiological pathways in human diseases, including CVDs, diabetes and cancer (9). Each miRNA has the potential to regulate multiple genes in biological processes, including development and metabolism $(8,9)$. Thus, miRNA dysregulation affects several pathological pathways in DM (9-11).

The aims of the present study were to assess the change in the miRNA expression profile in VSMCs following HG exposure, and to investigate the effect of dysregulated miRNA expression on VSMC proliferation and migration.

\section{Materials and methods}

Cell culture. VSMCs were isolated from thoracic aortic explants from male Sprague Dawley (SD) rats (age, 6 weeks; weight $200 \pm 20 \mathrm{~g}$ ) as previously described (12). The SD rats were purchased from Zhejiang Academy of Medical Science. The explants were cultured in low glucose DMEM (Thermo Fisher Scientific, Inc.) supplemented with 10\% FBS (Thermo Fisher Scientific, Inc.) and $1 \%$ antibiotic-antimycotic (Thermo Fisher Scientific, Inc.), and maintained at $37^{\circ} \mathrm{C}$ in a humidified atmosphere containing $5 \% \mathrm{CO}_{2}$ and $95 \%$ air. After 10 days, cells that had migrated from the aortic explants were collected and sub-cultured. Experiments were conducted from cell passages three to six. 293T cells were purchased from the American Type Culture Collection, and were maintained in high glucose DMEM containing $10 \%$ FBS and $1 \%$ antibiotic-antimycotic at $37^{\circ} \mathrm{C}$ in a humidified atmosphere containing $5 \% \mathrm{CO}_{2}$.

miRNA sequencing. VSMCs, normally cultured with $5.6 \mathrm{mM}$ glucose, were incubated with $22.2 \mathrm{mM}$ glucose (HG; Sigma-Aldrich; Merck KGaA) at $37^{\circ} \mathrm{C}$ for $48 \mathrm{~h}$. Total RNA was subsequently extracted from the HG-exposed and control VSMCs using TRIzol ${ }^{\circledast}$ reagent (Thermo Fisher Scientific, Inc.). Then, $\sim 1 \mu \mathrm{g}$ of the total RNA was used to prepare a small RNA library using a NEBNext Small RNA Library Prep Set (New England Biolabs, Inc.) according to the manufacturer's instructions. Single-end sequencing (50 bp) was performed using an Illumina HiSeq2500 system (Illumina, Inc.) according to the manufacturer's protocol. Raw reads were checked for contaminants and potential sequencing issues using FastQC (https://www.bioinformatics.babraham.ac.uk/projects/fastqc/; version 0.11.2). Low-quality reads, contaminating $5^{\prime}$ adapters and homopolymers were filtered. Reads were trimmed for $3^{\prime}$ adapters, and reads with a length of $<10$ and $>34$ nucleotide (nt) were discarded. Clean reads were processed using the Basic Local Alignment Search Tool (https://blast.ncbi.nlm. nih.gov/Blast.cgi; version 2.2.18). The Rfam10.1 database (https://rfam.org/) was used to annotate the RNA sequences and to align the sequences against miRNA precursors/mature miRNAs in the miRBase database (http://www.mirbase.org/; version 20.0) for the identification of known miRNAs. The read counts of each known miRNA were then normalized to the total counts of mapped sequence reads, and are presented as counts per million mapped reads. miRNAs with fold-changes $>1.5$ were selected as candidate miRNAs.

miRNA verification: Reverse transcription-quantitative PCR $(R T-q P C R)$. For further assessment of miRNA expression, miRNAs from the VSMCs in the aortic media were extracted using mirVana miRNA isolation kit (Thermo Fisher Scientific,
Inc.). cDNA was synthesized using the PrimeScript RT reagent kit (Takara Bio, Inc.) at $42^{\circ} \mathrm{C}$ for $1 \mathrm{~h}$ according to the manufacturer's instructions with specific rno-miR-125a RT primers (5'-GTCGTATCCAGTGCAGGGTCCGAGGTATTC GCACTGGATACGACAGGGAC-3'; Guangzhou RiboBio Co., Ltd.). qPCR was performed using the SYBR Premix Ex Taq kit (Takara Bio, Inc.) under the following conditions: Initial denaturation at $95^{\circ} \mathrm{C}$ for $30 \mathrm{sec}$, followed by 40 cycles at $95^{\circ} \mathrm{C}$ for $5 \mathrm{sec}$ and at $60^{\circ} \mathrm{C}$ for $30 \mathrm{sec}$ in an ABI Prism 7900 system (Applied Biosystems; Thermo Fisher Scientific, Inc.). The following sequences were used: rno-miR-125a forward, 5'-GCCGGGAGTGTCCAATTTCCCAGA-3' and reverse, 5'-CAGTGCAGGGTCCGAGGTAT-3'. U6 forward, 5'-CTC GCTTCGGCAGCACA-3' and reverse 5'-AACGCTTCACGA ATTTGCGT-3'. Relative miRNA expression was quantified using the $2^{-\triangle \Delta C q}$ method (13) and normalized to U6 expression.

miRNA, small interfering (si) RNA and plasmid transfection. siRNA against 3-hydroxy-3-methyglutaryl-coA reductase (HMGCR) and scrambled control (ctrl) siRNA were purchased from Shanghai GenePharma Co., Ltd. (cat. no. A10001). miR-125a mimic (sense, 5'-UCCCUGAGACCCUUUAAC CUGUGA-3' and antisense, 5'-UCACAGGUUAAAGGG UCUCAGGGA-3'), miRNA mimic negative control (miR-NC, sense, 5'-UUUGUACUACACAAAAGUACUG-3' and antisense, 5'-CAGUACUUUUGUGUAGUACAAA-3'), inhibitor (5'-UCACAGGUUAAAGGGUCUCAGGGA-3') and miRNA inhibitor negative control (miR-NC, 5'-CAGUACUUU UGUGUAGUACAAA-3') were purchased from Guangzhou RiboBio Co., Ltd. siRNA (100 $\mathrm{nM})$ and plasmid $(1 \mu \mathrm{g} / \mathrm{ml})$ transfections were performed using Lipofectamine 3000 (Thermo Fisher Scientific, Inc.). miRNA mimic (50 nM) and miRNA inhibitor $(50 \mathrm{nM})$ were transfected into VSMCs cultured under HG or normal conditions (5.6 $\mathrm{nM}$ glucose) by using Lipofectamine ${ }^{\circledR}$ RNAiMAX (Thermo Fisher Scientific, Inc.) according to the manufacturer's instructions. Western blotting and RT-qPCR analysis were used to detect the effects of gene silencing and miR-125a expression in VSMCs at $24 \mathrm{~h}$ after transfection (Fig. S1).

VSMC proliferation assay. VSMCs were cultured in different concentrations of glucose $(5.6,11.1,22.2$ and $44.5 \mathrm{mM})$ at $37^{\circ} \mathrm{C}$ in a humidified atmosphere containing $5 \% \mathrm{CO}_{2}$ for $72 \mathrm{~h}$ and cell proliferation was detected with a Cell Counting Kit-8 (CCK-8) assay (Dojindo Molecular Technologies, Inc.). In addition, after $24 \mathrm{~h}$ transfection with HMGCR siRNA or miR-125a mimic, VSMCs were plated at a density of $\sim 5,000$ cells/well in 96 -well plates and maintained in HG-culture medium $(22.2 \mathrm{mM})$ for $72 \mathrm{~h}$. Cell proliferation was also determined using the CCK-8 assay at $37^{\circ} \mathrm{C}$ for $3 \mathrm{~h}$ and the absorbance was measured at $450 \mathrm{~nm}$ according to the manufacturer's instructions.

Wound healing assay. VSMCs were plated in 6-well plates at a density of $5 \times 10^{5}$ cells per well and cultured in different concentrations of glucose (5.6, 11.1, 22.2 and $44.5 \mathrm{mM}$ ). At $90 \%$ confluency, cells were exposed to mitomycin C $(10 \mathrm{mg} / \mathrm{ml}$; Sigma-Aldrich; Merck KGaA), a potent cell proliferation inhibitor, at $37^{\circ} \mathrm{C}$ for $2 \mathrm{~h}$. The confluent cell monolayer was subsequently scratched using a $200 \mu \mathrm{l}$ pipette tip. After wounding, the medium was replaced with fresh serum-free 
medium. Serial images were obtained immediately after wounding $(0 \mathrm{~h})$ and after $48 \mathrm{~h}$ by light microscopy (magnification, $\mathrm{x} 20$ ). VSMC migration was determined by calculating the initial wound area and the wound area after $48 \mathrm{~h}$, the percent wound size (the wound area at $48 \mathrm{~h}$ relative to the initial wound area) was analyzed by ImageJ software (version 1.46r; National Institutes of Health).

Dual-luciferase assay. The 3'-UTR of HMGCR carrying the predicted miR-125a-binding site (http://www.targetscan. org/vert_72/; version 7.2) was amplified and subcloned into the pmirGLO vector (Promega Corporation) to generate the wild-type (WT) HMGCR dual-luciferase expression vector (HMGCR-WT). The rapid mutation kit (New England Biolabs, Inc.; cat. no. E0554S) was used to generate a HMGCR mutant (Mut), which was also cloned into the pmirGLO vector (HMGCR-Mut). 293T cells were co-transfected with HMGCR-Mut or HMGCR-WT, and miR-125a or the control mimics using Lipofectamine ${ }^{\circledR} 3000$ (Thermo Fisher Scientific, Inc.). After $48 \mathrm{~h}$, the activities of firefly and Renilla luciferase were determined using a Dual-Luciferase Reporter assay system (Promega Corporation). The results were shown as the ratio of Renilla to firefly luciferase activity.

Western blot analysis. Aortic media samples from SD rats or VSMCs were lysed with RIPA lysis buffer (Beyotime Institute of Biotechnology) and $1 \%$ protease inhibitors. Bicinchoninic acid assay kit (Thermo Fisher Scientific, Inc.) was used to quantify the protein samples. Western blot analysis was carried out according to standard procedures. Proteins (30 $\mu \mathrm{g} / \mathrm{lane})$ were separated via SDS-PAGE on a $10 \%$ gel and then transferred to a $0.22-\mu \mathrm{m}$ PVDF membrane. The primary antibodies used included anti-HMGCR (1:1,000; cat. no. ab174830; Abcam), anti-farnesyl diphosphate synthase (FDPS; 1:1000; cat. no. ab189874; Abcam), anti-squalene synthase (SQS; 1:1,000; cat. no. ab195046; Abcam), anti-geranylgeranyltransferase type I (GGTase-I; 1:1,000; cat. no. ab122122; Abcam) and anti-GAPDH $(1: 3,000$; cat. no. ET1601-4; Huabio) were incubated at room temperature for $3 \mathrm{~h}$. A horseradish peroxidase-conjugated goat anti-rabbit IgG H\&L (1:3,000; cat. no. ab7090; Abcam) was used as the secondary antibody and was incubated at room temperature for $1 \mathrm{~h}$. Protein bands were visualized using enhanced chemiluminescence and a ChemiScope Western Blot Imaging system (Clinx Science Instruments Co., Ltd.). The gray-scale value was analyzed using Image J software (version 1.46r; National Institutes of Health) for densitometry analysis.

Animal experiments. A total of 60 male SD rats (age, 6-week old; weight, $200 \pm 20 \mathrm{~g}$ ) were purchased from the Shanghai Laboratory Animal Center. In total, 24 rats were used for the control group and 36 rats for the DM group. Animals had free access to food and water, and were housed in a pathogen-free environment with normoxic atmosphere (temperature, $20-25^{\circ} \mathrm{C}$ ). The animal experiments were reviewed and approved by The Institutional Animal Care and Use Committee of Zhejiang University. DM was induced by daily intraperitoneal injection of $50 \mathrm{mg} / \mathrm{kg}$ streptozotocin (STZ; Sigma-Aldrich; Merck KGaA) for 5 days after a 4-h fast. Control rats received an intraperitoneal injection of vehicle $(0.1 \mathrm{M}$ citrate buffer;
pH 4.5; $0.1 \mathrm{ml})$. Fasting plasma glucose (FPG) levels were measured in $0.05 \mathrm{ml}$ venous blood drawn from the caudal vein using a glucometer (ACCU-CHECK Active kit; Roche Diagnostics). At 3 days after the last STZ injection, rats with FPG level $>16.7 \mathrm{mM}$ were considered to have been successful were established as DM models (14). The 12 rats failed to induce DM were not used for subsequent experiments.

Blood lipid analysis. At the start and then 5, 10 and 20 weeks after STZ injection, serum total cholesterol (cat. no. 05168538190), high- and low-density lipoprotein cholesterol (cat. nos. 07528582190 and 07005768190 ), triglyceride concentration (cat. no. 05171407190) and FPG (cat. no. 05168791190) in $3 \mathrm{ml}$ blood samples were collected from the inferior vena cava of the anesthetized animal following overnight fasting conditions, and were determined using commercially available kits (Sigma-Aldrich; Merck KGaA). Fasting serum insulin (FINS) was determined by chemiluminometry (15). The following equation: (FINS in $\mathrm{mU} / 1 \times \mathrm{FPG}$ in $\mathrm{mmol} / \mathrm{l}) / 22.5$, was used to assess the homeostasis model of insulin resistance, while the homeostasis model of $\beta$-cell function was quantified as follows: (FINS in $\mathrm{mU} / 1$ x 20)/(FPG in mmol/l-3.5) (15).

Histological analysis. The aorta was dissected in situ from the ascending aorta to the iliac bifurcation. Peripheral fat was removed under an anatomical microscope and the aorta was subsequently fixed using $10 \%$ neutral formalin for $24 \mathrm{~h}$ at room temperature and embedded in paraffin at room temperature for $6 \mathrm{~h}$. Embedded specimens were cut into $5 \mu \mathrm{m}$ sections, and subjected to standard hematoxylin and eosin staining (hematoxylin, $10 \mathrm{~min}$; eosin, $30 \mathrm{sec}$; room temperature) to determine the media thickness (MT) and media cross-sectional area (MCSA), which is the area between the internal and external elastic lamina. Morphometric analysis was performed using Image-Pro Plus software (version 6.0) (12).

Statistical analysis. The differences between two groups were compared using the Student's t-test. One-way ANOVA followed by Bonferroni post-hoc test was used to determine significant differences between multiple groups. All the experiments were repeated $\geq 3$ times, and data are presented as the mean $\pm \mathrm{SD}$. $\mathrm{P}<0.05$ was considered to indicate a statistically significant difference.

\section{Results}

$H G$-induced proliferation and migration of VSMCs is consistent with decreased miR-125a expression. CCK-8 assay results indicated that VSMCs cultured in different concentrations of glucose $(5.6,11.1,22.2$ and $44.5 \mathrm{mM})$ had increased proliferation (Fig. 1A) and migration (Fig. 1B and C) in a dose-dependent manner. To determine whether HG exposure altered miRNA expression in VSMCs, the miRNA expression profile of VSMCs exposed to HG (22.2 mM glucose) for $48 \mathrm{~h}$ was compared with VSMCs cultured under normal conditions (5.6 mM glucose). It was found that a total of 42 miRNAs were differentially expressed (fold-change, $>1.5$ ) between the two groups. Of the 42 miRNAs, 30 miRNAs were upregulated, including rno-miR-431, rno-miR-182, rno-miR-708-5p, 

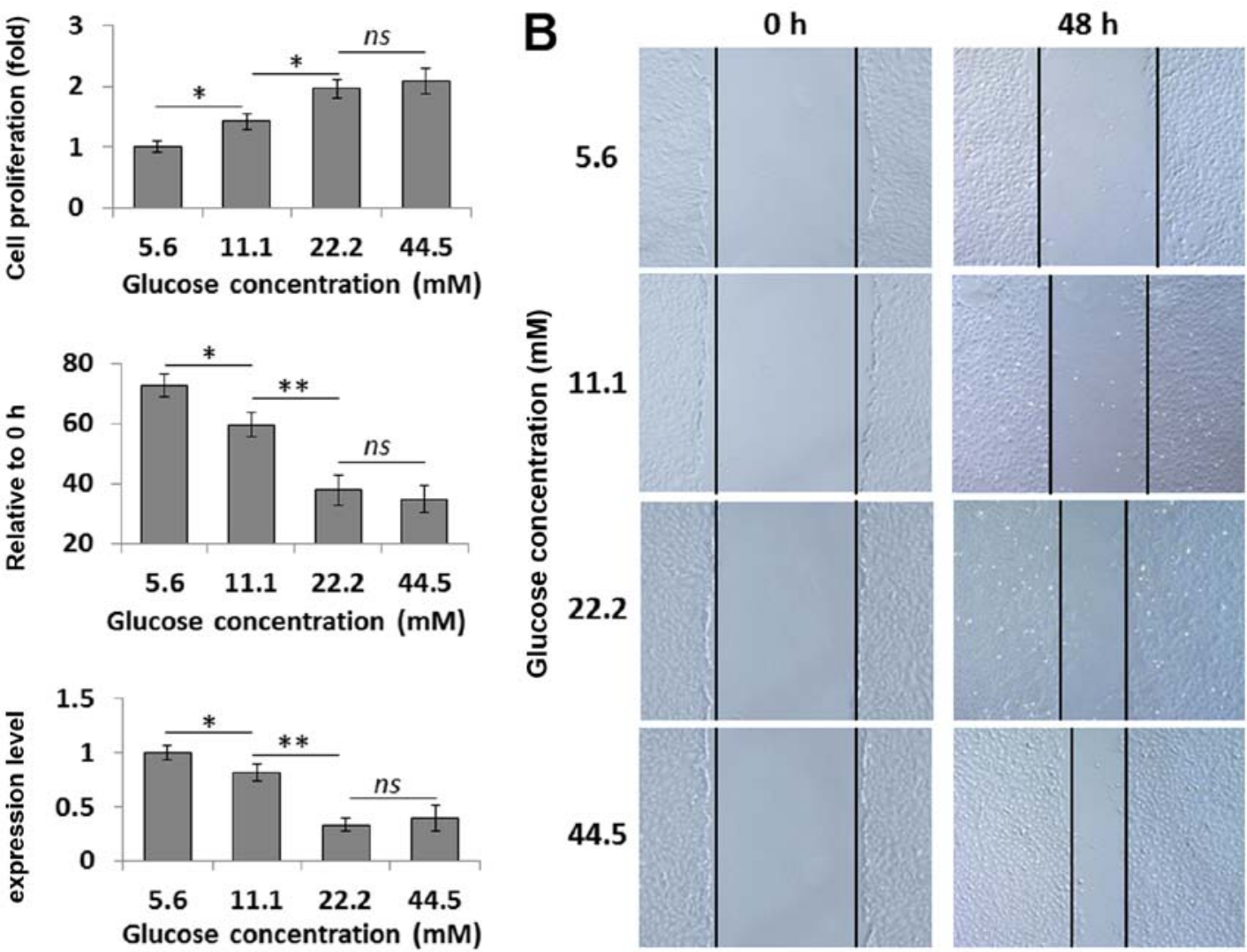

Figure 1. miR-125a expression is inversely related to HG-induced VSMC proliferation and migration. (A) VSMCs were incubated with different concentrations of glucose $(5.6,11.1,22.2$ and $44.5 \mathrm{mM}$ ) for $48 \mathrm{~h}$. Cell proliferation was measured using the Cell Counting Kit- 8 assay. (B) A wound healing assay was performed to investigate the (C) migration of HG treated-VSMCs (magnification, x20). Cell proliferation was inhibited using mitomycin C before wound scratching. (D) Expression of miR-125a in HG-treated VSMCs was analyzed by reverse transcription-quantitative PCR. Data are presented as the mean \pm SD. $\mathrm{n}=3 .{ }^{*} \mathrm{P}<0.05,{ }^{* *} \mathrm{P}<0.01 ; \mathrm{ns}$, not significant; miR, microRNA; HG, high glucose; VSMCs, vascular smooth muscle cells.

rno-miR-495 and rno-miR-377-3p, and 12 miRNAs were downregulated, including rno-miR-125a-5p, rno-miR-331-3p, rno-miR-450b-5p, rno-miR-101a-3p and rno-miR-125b-5p, in VSMCs exposed to HG (Table SI). Among the differentially expressed miRNAs, miR-125a exhibited the largest change. Furthermore, it was demonstrated that glucose stimulation decreased the expression of miR-125a in a dose-dependent manner in VSMCs (Fig. 1D).

miR-125a abrogates HG-induced VSMC migration and proliferation. To investigate whether HG-induced VSMC proliferation and migration were dependent on decreased miR-125 expression, a miR-125a mimic was used to overexpress miR-125a in VSMCs. It was found that exposure to $22.2 \mathrm{mM}$ glucose significantly increased VSMC proliferation compared with the control (Fig. 2A). Moreover, this increased proliferation was partially suppressed following miR-125a mimic transfection, and was not affected by miR-NC transfection. In addition, wound healing assay results indicated that HG-treated VSMCs exhibited increased migration, while transfection with a miR-125a mimic reduced the migration of HG-treated VSMCs, which was determined by the percent wound size (the wound area at $48 \mathrm{~h}$ relative to the initial wound area; Fig. 2B and C).

HMGCR is a direct target of miR-125a. HMGCR was predicted as a candidate target of miR-125a using TargetScan software (http://www.targetscan.org/vert_72/; version 7.2), and it was identified that the HMGCR 3'-UTR had a potential binding site for miR-125a (Fig. 3A). To investigate this, HMGCR-WT and HMGCR-Mut were subcloned into the pmirGLO vectors, and the resulting plasmids were co-transfected with a miR-125a mimic or miR-NC. Compared with the miR-NC, the miR-125a mimic significantly reduced the luciferase activity of HMGCR-WT. Transfection with the HMGCR-Mut abrogated the inhibitory effect of miR-125a (Fig. 3B). Moreover, transfection with a miR-125a mimic and miR-125a inhibitor significantly reduced and increased HMGCR protein expression levels in VSMCs, respectively (Fig. 3C). Therefore, the present results indicated that HMGCR may be a direct target of miR-125a.

HMGCR downregulation suppresses HG-induced VSMC proliferation and migration. The effects of HMGCR on HG-induced cell proliferation and migration were evaluated by knocking down HMGCR expression in VSMCs, using an HMGCR-specific siRNA. It was found that the expression of HMGCR in VSMCs was upregulated in a dose-dependent manner following $\mathrm{HG}$ exposure (Fig. 4A). Furthermore, CCK-8 and wound healing assays demonstrated that HG-induced proliferation and migration of VSMCs were significantly repressed by transfection with si-HMGCR, compared with transfection with si-Ctrl (Fig. 4B-D). Collectively, the present results suggested that HG-induced VSMC proliferation and 
A
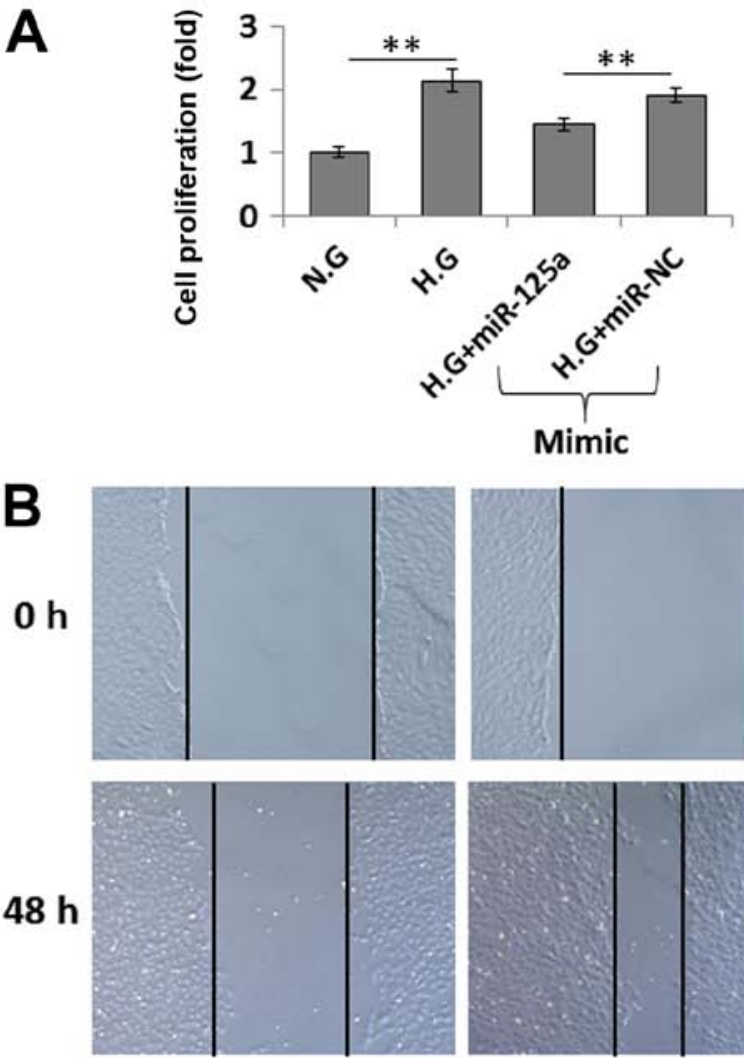

N.G
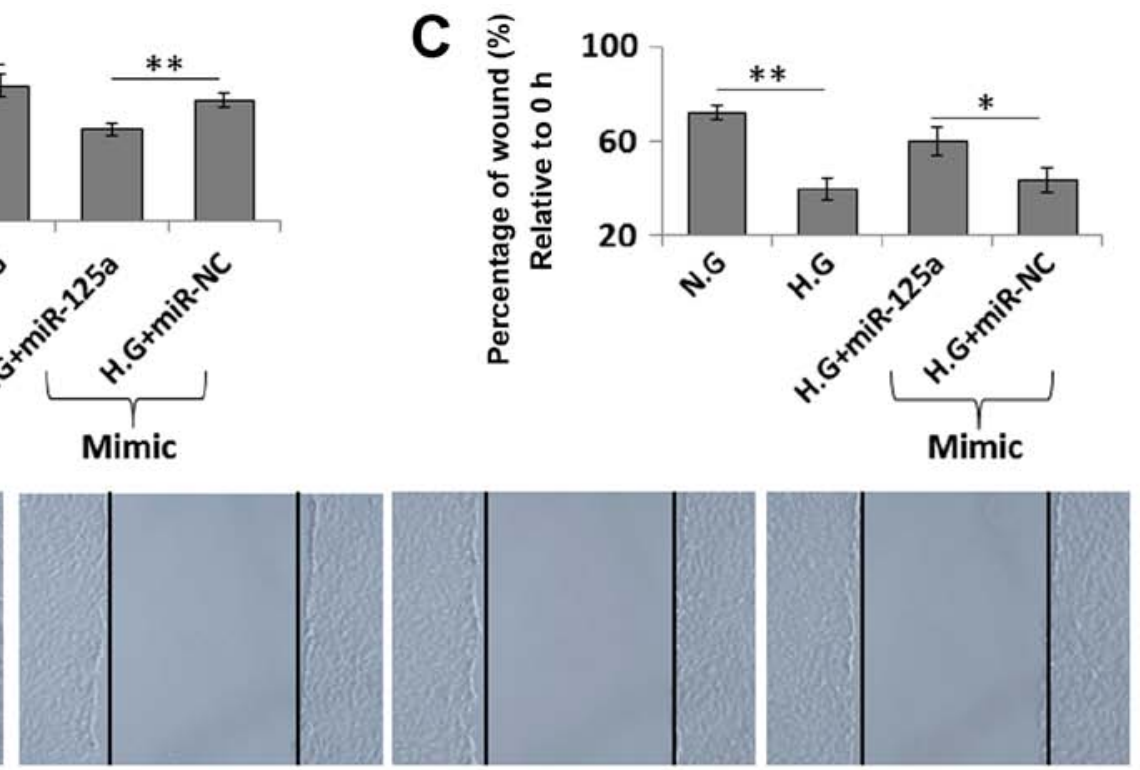

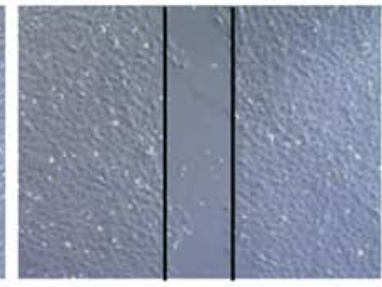

H.G

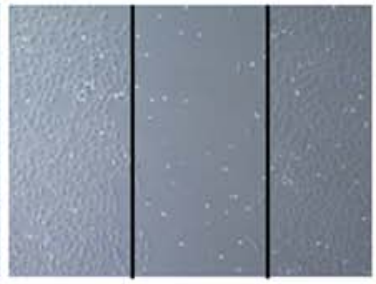

H.G + miR-125a

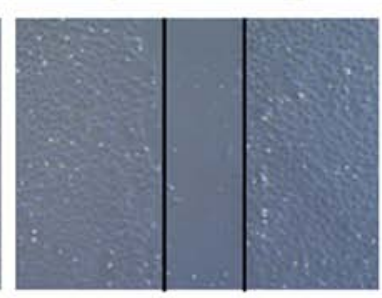

H.G + miR-NC

Figure 2. Transfection with miR-125a abrogates HG-induced VSMC proliferation and migration. (A) Cell Counting Kit-8 assay results demonstrated that transfection with a miR-125a mimic reduced HG-induced VSMC proliferation. (B) A wound healing assay (C) showed that miR-125a transfection reduced HG-induced VSMC migration. Magnification, $\mathrm{x} 20$. Cell proliferation was inhibited by using mitomycin $\mathrm{C}$ before wound scratching. Data are presented as the mean \pm SD. $n=3$. ${ }^{*} \mathrm{P}<0.05,{ }^{* *} \mathrm{P}<0.01$; ns, not significant; miR, microRNA; H.G, high glucose; N.G, normal glucose; VSMC, vascular smooth muscle cell; NC, negative control.

A

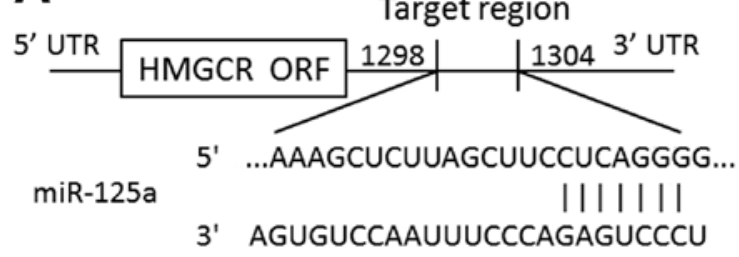

B

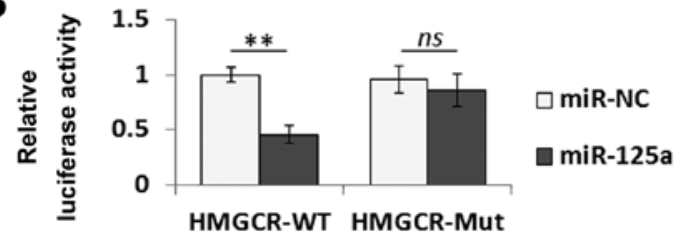

C

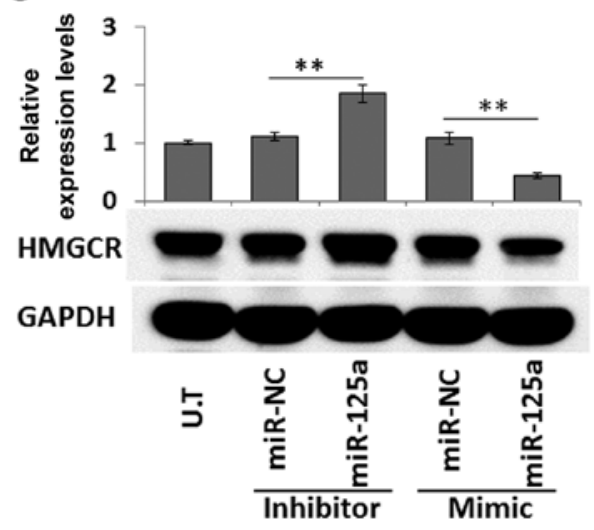

Figure 3. Validation of HMGCR as a direct target of miR-125a. (A) Target site of miR-125a in the HMGCR 3'-UTR. (B) Dual-luciferase activity of the WT and Mut HMGCR 3'-UTR reporter in the presence of miR-125a or miR-NC. (C) Western blot analysis of HMGCR expression in VSMCs transfected with a miR-125a mimic, miR-125a inhibitor or miR-NC. Data are presented as the mean \pm SD. $n=3$. ${ }^{* *} \mathrm{P}<0.01$; ns, not significant; HMGCR, 3-hydroxy-3-methylglutaryl-coenzyme A reductase; miR, microRNA; WT, wild-type; Mut, mutant; 3'-UTR, 3'-untranslated region; NC, negative control; VSMC, vascular smooth muscle cell; UT, untreated VSMCs.

migration may be due to increased HMGCR expression, and decreased miR-125a expression.

miR -125 a regulates the mevalonate signaling pathway via HMGCR in VSMCs. HMGCR is one of the key enzymes in the mevalonate pathway, which is associated with development of atherosclerosis (10). To further determine the mechanistic role of miR-125a in the progression of atherosclerosis, the effect of miR-125a on the mevalonate signaling pathway was evaluated. Key enzymes in this pathway, including FDPS and GGTase-I were upregulated, while SQS was downregulated, in VSMCs exposed to $\mathrm{HG}$, but the dysregulation of these enzymes could 

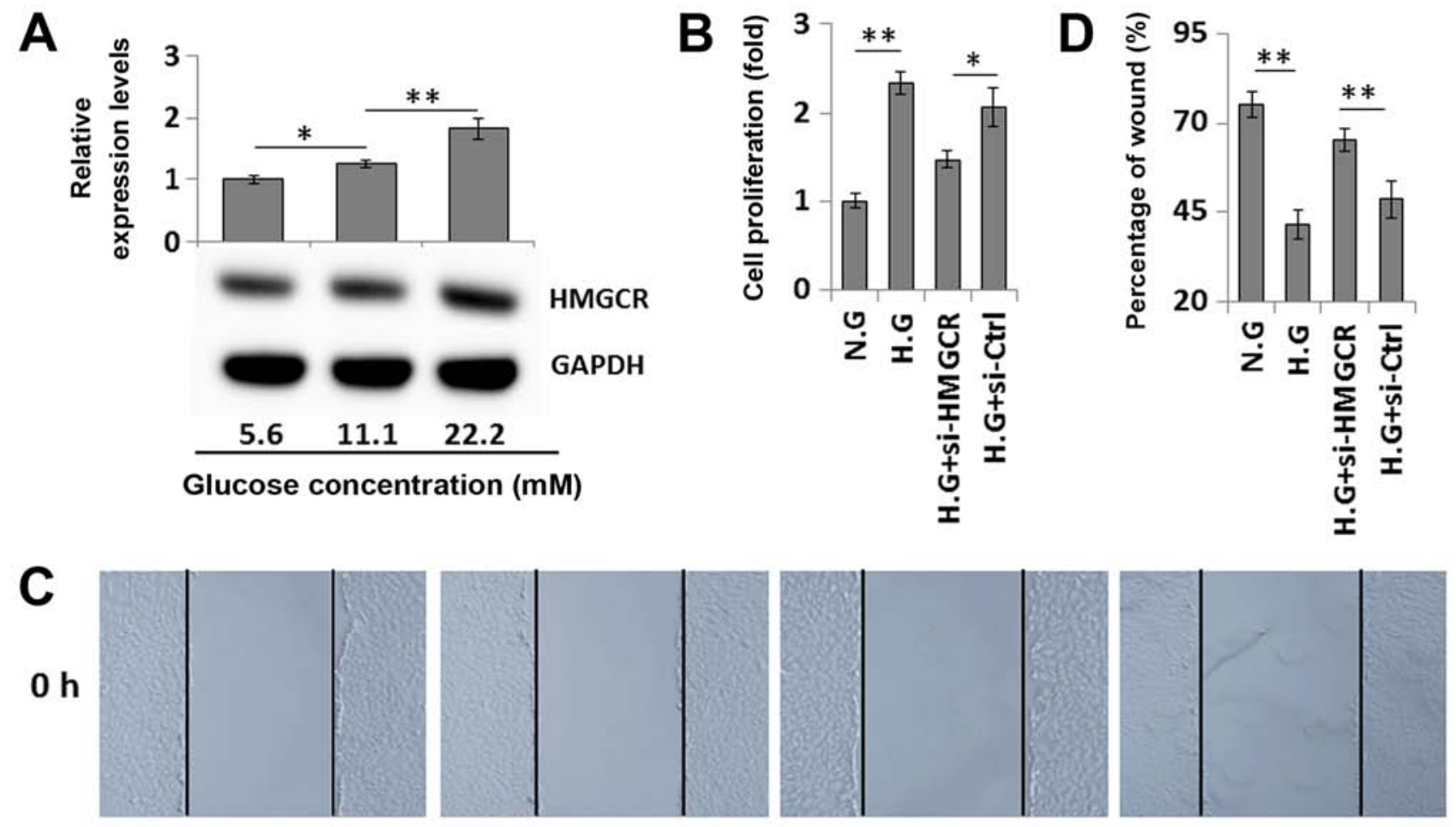

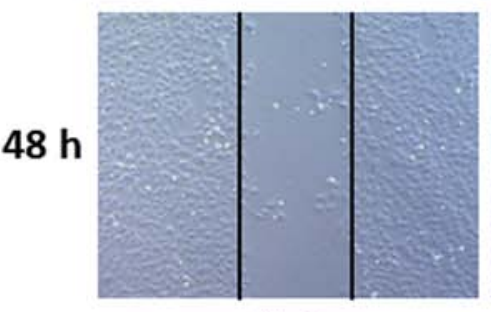

N.G

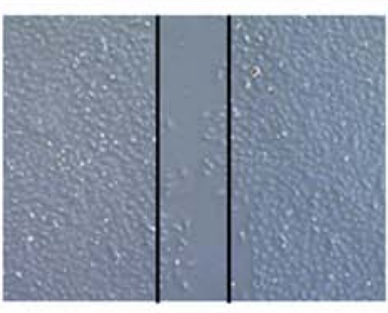

H.G

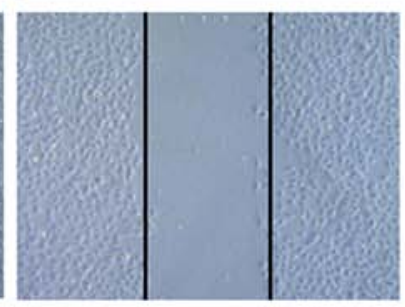

H.G + si-HMGCR

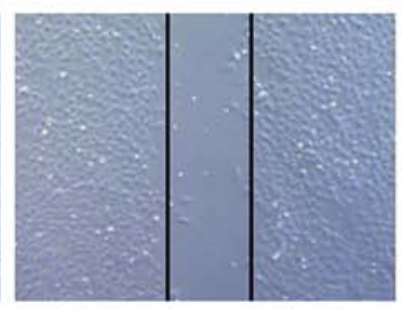

H.G + si-Ctrl

Figure 4. HMGCR is involved in HG-induced VSMC proliferation and migration. (A) Western blot analysis of HMGCR expression in VSMCs exposed to HG. (B) Cell Counting Kit-8 assay results found that downregulation of HMGCR reduced the HG-induced proliferation of VSMCs. (C) Wound healing assay (D) identified that downregulation of HMGCR reduced the HG-induced migration of VSMCs. Magnification, x20. Cell proliferation was inhibited by using mitomycin $\mathrm{C}$ before wound scratching. Data are presented as the mean $\pm \mathrm{SD}$. n=3. ${ }^{*} \mathrm{P}<0.05,{ }^{* *} \mathrm{P}<0.01$. ns, not significant; HMGCR, 3-hydroxy-3-methylglutaryl-coenzyme A reductase; H.G, high glucose; VSMC, vascular smooth muscle cell; N.G, normal glucose; Ctrl, control; siRNA, small interfering RNA.

be significantly reversed following transfection with the miR-125a mimic (Fig. 5A). However, miR-125a inhibition could upregulate the FDPS and GGTase-I levels and downregulate the SQS levels in VSMCs, even under normal culture conditions (Fig. 5B). Therefore, the present results indicated that miR-125a may be involved in HG-induced dysregulation of the mevalonate signaling pathway by targeting HMGCR.

miR-125a-mediated regulation of the mevalonate signaling pathway contributes to HG-induced atherosclerosis. To further investigate the effect of miR-125a on the mevalonate signaling pathway in vivo, a rat STZ-induced model of DM was established. It was demonstrated that blood glucose levels gradually increased over 1 week in the animal model. Moreover, the elevation of blood glucose was consistent with the development of aortic atherosclerosis (Table I), which was determined by increased MT and MCSA levels in the thoracic aorta in experimental rats compared with age-matched controls (Fig. 6A). The expression levels of miR-125a, HMGCR, FDPS, SQS and GGTase-I in the aortic media were subsequently analyzed. It was found that miR-125a expression was gradually downregulated, and the protein expression levels of HMGCR, FDPS, SQS and GGTase-I were dysregulated in the aortic media of the experimental rats compared with age-matched controls after 5 weeks (Fig. 6B and C). Thus, miR-125a-mediated dysregulation of the mevalonate signaling pathway may be involved in hyperglycemia-associated atherosclerosis.

\section{Discussion}

VSMCs maintain the physiological structure of blood vessels and play a key role in the development of atherosclerosis (10). Moreover, VSMCs are able to switch phenotypes by undergoing differentiation or dedifferentiation, which is closely related to the formation of atherosclerosis (10). Pathological states, such as hyperglycemia in DM, may cause VSMCs to enter a synthetic state, which is characterized by excessive proliferation, migration and extracellular matrix secretion $(4,9)$. In atherosclerosis, the VSMC phenotype contributes to a series of pathological processes relevant to CVD, including plaque formation (10). Therefore, the inhibition of hyperglycemia-induced vascular dysfunction may provide a novel strategy for the treatment of 

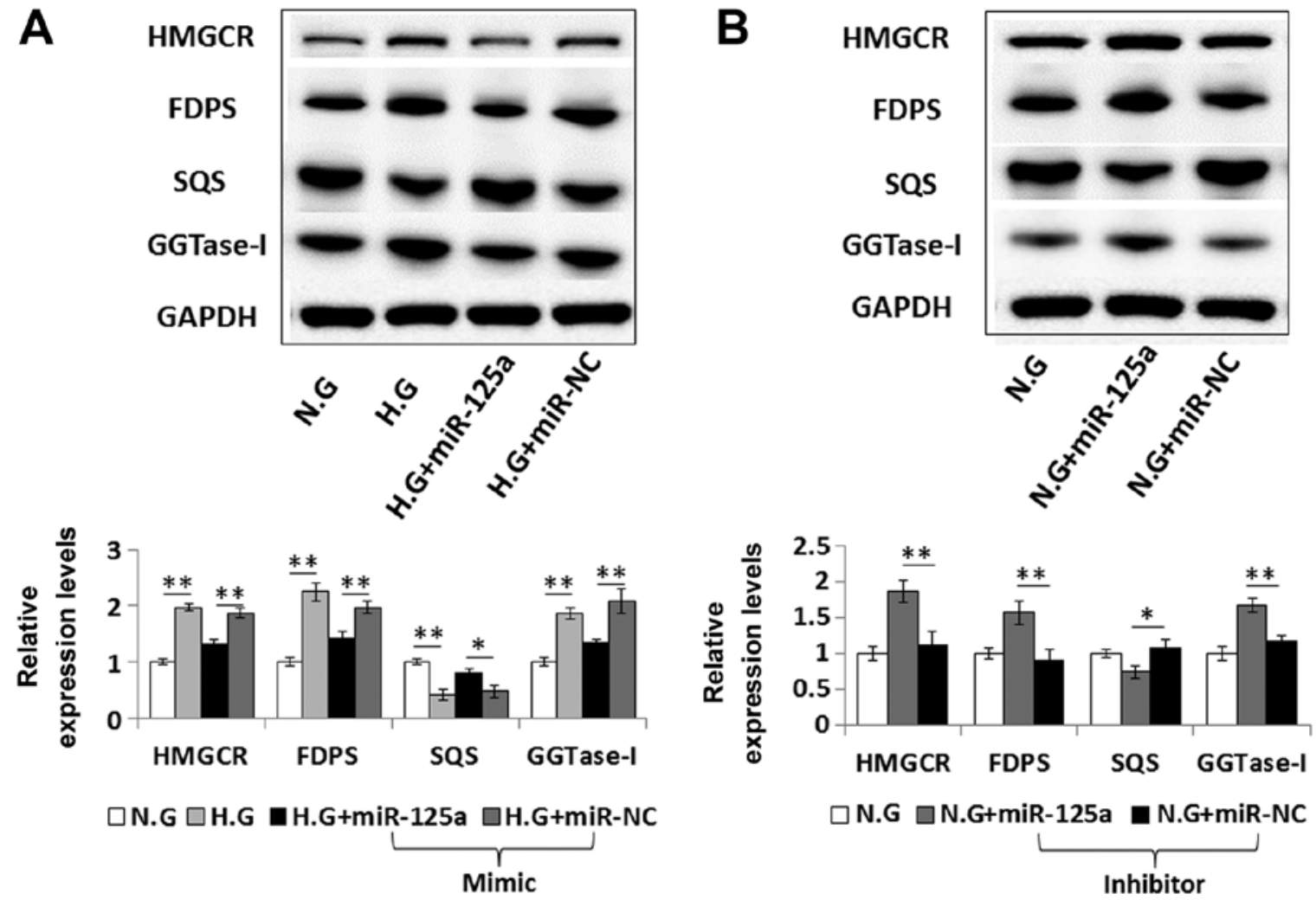

Figure 5. miR-125a regulates the expression levels of key enzymes in the mevalonate signaling pathway. (A) Protein expression levels of HMGCR, FDPS, SQS and GGTase-I were measured by western blotting. miR-125a mimic transfection reversed HG-induced dysregulation of these proteins in VSMCs. (B) miR-125a inhibitor transfection induced the activation of the mevalonate signaling pathway in VSMCs cultured under normal conditions (5.6 nM glucose). Data are presented as the mean \pm SD. $\mathrm{n}=3 .{ }^{*} \mathrm{P}<0.05,{ }^{* *} \mathrm{P}<0.01$. ns, not significant; miR, microRNA; HMGCR, 3-hydroxy-3-methylglutaryl-coenzyme A reductase; FDPS, farnesyl diphosphate synthase; SQS, squalene synthase; GGTase-I, geranylgeranyltransferase type I; H.G, high glucose; N.G, normal glucose; VSMC, vascular smooth muscle cell; NC, negative control.

DM-associated CVD (12). The present results suggested that HG-induced VSMC proliferation and migration were associated with decreased miR-125a expression and dysregulated expression of mevalonate pathway-related enzymes. Moreover, miR-125a upregulation abrogated HG-induced VSMC proliferation and migration by targeting HMGCR, and modulating the mevalonate signaling pathway.

miRNAs play important roles in physiological and pathological conditions $(16,17)$. Furthermore, previous studies have shown that several miRNAs are involved in the regulation of cardiovascular physiological and pathological processes, and are closely related to VSMCs (18-23). miR-21 promotes neointima formation following vascular injury by increasing VSMC proliferation, which occurs via the inhibition of phosphatase and subsequent activation of the PI3K/Akt signaling pathway (20). Moreover, miR-221, miR-222, miR-26a and miR-138 promote VSMC proliferation or migration by inhibiting cyclin-dependent kinase inhibitor $1 \mathrm{~B}$, cyclin-dependent kinase inhibitor 1C, SMAD family member 1 and sirtuin 1 , respectively (21-23). However, it has been revealed that miR-143/145 and miR-133 decrease VSCM proliferation and promote the synthetic phenotype $(24,25)$. miR-24 and miR-145 also decrease VSMC proliferation and migration by targeting HMGB1 and Rho-associated coiled-coil containing protein kinase 1 (ROCK1). In addition, ROCK1 promotes the proliferation and migration of VSMCs by acting on Kruppel like factor 4 via miR-92a $(26,27)$.
The present study used bioinformatics analysis and RT-qPCR to demonstrate that miR-125a may modulate HG-induced proliferation and migration of VSCMs. miR-125a is an endogenous non-coding miRNA with regulatory functions that are conserved from nematodes to humans (28). Moreover, miR-125a serves important roles in several cellular processes, including cell differentiation, hematopoiesis, organogenesis, cell proliferation, lipid metabolism and apoptosis, by targeting transcription factors, matrix-metalloproteases and growth factors $(28,29)$. The tissue expression level of miR-125a is typically upregulated during differentiation, but is often downregulated in disease states, including diabetic retinopathy and breast cancer (30). Therefore, dysregulated miR-125a expression may be involved in the progression of diseases and can serve as a potential therapeutic target $(31,32)$. The present results suggested that miR-125a expression was downregulated in VSMCs exposed to HG and in aortic media from rats with STZ-induced DM. Furthermore, it was found that miR-125a mimic transfection reversed $\mathrm{HG}$-induced VSMC proliferation and migration. Therefore, it was speculated that decreased miR-125a expression may contribute to the VSMC phenotypic switch in atherosclerosis.

To further investigate the role of miR-125a in HG-induced VSMC proliferation and migration, potential targets of miR-125a were identified using miRNA target prediction software. It was found that HMGCR was a target gene of miR-125a, and a luciferase reporter assay was subsequently 
Table I. Biochemical characteristics of rat model.

\begin{tabular}{|c|c|c|c|c|c|c|}
\hline Weeks & FPG (mM) & TC (mM) & LDL-C (mM) & Insulin (IU/1) & HOMA-IR & HOMA-B \\
\hline \multicolumn{7}{|l|}{ Control rats } \\
\hline Initial $(n=6)$ & $6.71 \pm 0.29$ & $2.93 \pm 0.10$ & $1.48 \pm 0.03$ & $5.79 \pm 0.63$ & $1.72 \pm 0.24$ & $36.08 \pm 4.19$ \\
\hline $5(n=6)$ & $6.32 \pm 0.26$ & $2.79 \pm 0.11$ & $1.32 \pm 0.07$ & $7.02 \pm 0.84$ & $1.91 \pm 0.27$ & $39.02 \pm 3.01$ \\
\hline $10(n=6)$ & $7.40 \pm 0.58$ & $2.53 \pm 0.07$ & $1.63 \pm 0.09$ & $6.61 \pm 0.90$ & $2.01 \pm 0.42$ & $33.98 \pm 3.15$ \\
\hline $20(n=6)$ & $7.84 \pm 0.84$ & $2.83 \pm 0.14$ & $1.61 \pm 0.12$ & $6.99 \pm 0.74$ & $2.36 \pm 0.12$ & $41.65 \pm 4.43$ \\
\hline \multicolumn{7}{|l|}{ Diabetic rats } \\
\hline Initial $(n=6)$ & $7.27 \pm 0.31$ & $2.91 \pm 0.16$ & $1.47 \pm 0.16$ & $5.87 \pm 0.76$ & $1.90 \pm 0.40$ & $31.14 \pm 3.36$ \\
\hline $5(n=6)$ & $20.22 \pm 0.43^{\mathrm{b}}$ & $3.97 \pm 0.15^{\mathrm{a}}$ & $1.70 \pm 0.04^{\mathrm{a}}$ & $8.72 \pm 0.93$ & $7.84 \pm 1.18^{\mathrm{b}}$ & $10.43 \pm 2.02^{b}$ \\
\hline $10(n=6)$ & $24.67 \pm 2.91^{\mathrm{b}}$ & $4.37 \pm 0.09^{b}$ & $2.03 \pm 0.07^{\mathrm{b}}$ & $4.65 \pm 0.70^{\mathrm{a}}$ & $5.10 \pm 1.10^{\mathrm{b}}$ & $4.39 \pm 0.54^{b}$ \\
\hline $20(n=6)$ & $27.08 \pm 3.43^{\mathrm{b}}$ & $4.93 \pm 0.14^{\mathrm{b}}$ & $2.17 \pm 0.12^{b}$ & $2.13 \pm 1.07^{\mathrm{b}}$ & $2.56 \pm 0.82$ & $1.81 \pm 0.22^{\mathrm{b}}$ \\
\hline
\end{tabular}

Data are presented as the mean $\pm \mathrm{SD} .{ }^{\mathrm{a}} \mathrm{P}<0.05,{ }^{\mathrm{b}} \mathrm{P}<0.01 \mathrm{vs}$. age-matched control rats. FPG, fasting plasma glucose; TC, total cholesterol; LDL-C, Low-density lipoprotein cholesterol; HOMA-IR, homeostasis model assessment of insulin resistance; HOMA-B, homeostasis model assessment of $\beta$-cell function.

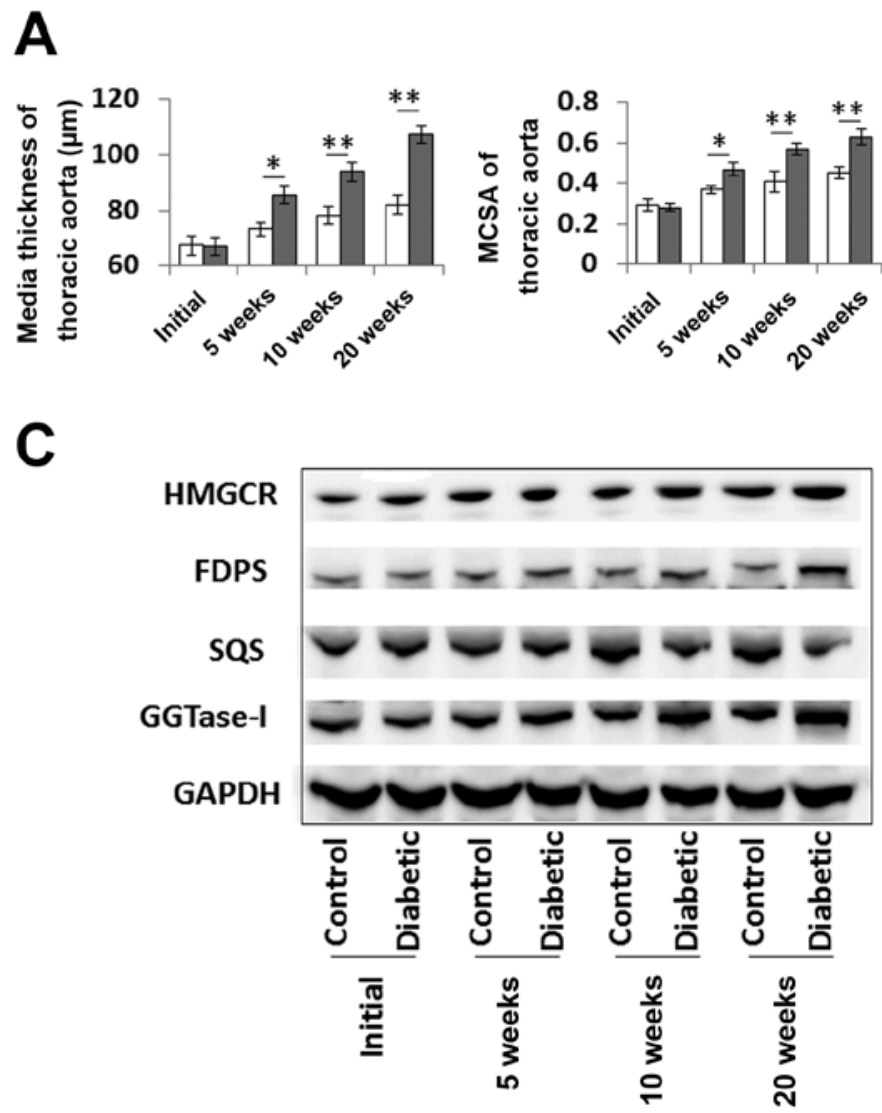

B
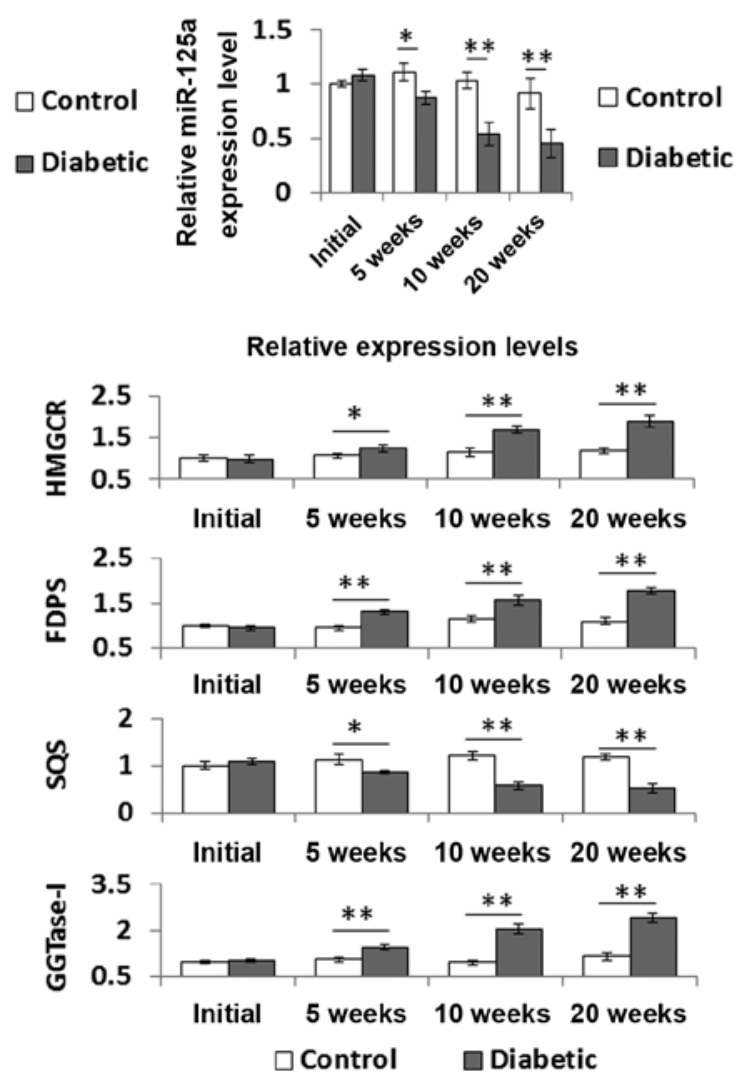

Figure 6. High glucose-induced atherosclerosis is associated with miR-125a-mediated dysregulation of the mevalonate signaling pathway. (A) Media thickness and MCSA of the thoracic aorta from Sprague Dawley rats at time 0, and 5, 10 and 20 weeks after streptozotocin injection. (B) miR-125a expression in the aortic media. (C) Protein expression levels of HMGCR, FDPS, SQS and GGTase-I in the aortic media were measured by western blotting. Data are presented as the mean \pm SD. $n=6 .{ }^{*} \mathrm{P}<0.05,{ }^{* *} \mathrm{P}<0.01$. ns, not significant; MCSA, media cross-sectional area; HMGCR, 3-hydroxy-3-methylglutaryl-coenzyme A reductase; FDPS, farnesyl diphosphate synthase; SQS, squalene synthase; GGTase-I, geranylgeranyltransferase type I; miR, microRNA.

used to assess this observation. Moreover, miR-125a-mediated regulation of HMGCR was further demonstrated by western blot analysis. It was demonstrated that the expression of HMGCR in VSMCs was decreased following transfection with a miR-125a mimic, but increased following transfection with a miR-125a inhibitor. Furthermore, the downregulation of HMGCR expression abrogated HG-induced proliferation and migration of VSCMs, similarly to the effects observed 
following miR-125a mimic transfection. Collectively, the present results suggested that HMGCR is a functional target gene of miR-125a in VSMCs, and that miR-125a may decrease the progression of atherosclerosis by targeting HMGCR.

HMGCR is an important enzyme in the mevalonate signaling pathway, which is a metabolic pathway that synthesizes isoprene pyrophosphate and dimethylallyl pyrophosphate from acetyl coenzyme A (33). The end-product of this pathway is an activated isoprene unit that serves as precursor of steroids, terpenoids and other biological molecules $(33,34)$. Previous studies $(12,35,36)$ have revealed that the mevalonate signaling pathway serves an important role in the cardiovascular system. It has been shown that the expression levels of key enzymes in the mevalonate pathway, including HMGCR, FDPS, SQS and GGTase-1, are significantly dysregulated in aortas from spontaneously hypertensive rats or mice with STZ-induced DM, and are associated with aortic structural remodeling or atherosclerosis development, respectively $(10,35)$. The present results indicated that dysregulated expression levels of key enzymes in the mevalonate signaling pathway may accelerate atherosclerosis in DM. Moreover, the dysregulated expression levels of HMGCR, FDPS, SQS and GGTase-I were associated with gradually downregulated miR-125a expression in the aortic media from rats with STZ-induced DM, or in cultured VSMCs exposed to HG. Thus, miR-125a may be involved in the HG-induced dysregulation of the mevalonate signaling pathway. However, it was identified that HG-induced activation of the mevalonate signaling pathway in VSMCs was suppressed following miR-125a mimic transfection.

In conclusion, it was found that downregulation of miR-125a expression was associated with HG-induced VSMC proliferation and migration. Furthermore, upregulation of miR-125a abrogated these effects by directly targeting HMGCR and suppressing the activation of the mevalonate signaling pathway. Therefore, miR-125a-mediated regulation of this pathway may serve as a potential therapeutic strategy for DM-related atherosclerosis.

\section{Acknowledgements}

Not applicable.

\section{Funding}

This study was supported by National Natural Science Funds (grant nos. 81500616 and 81701365).

\section{Availability of data and materials}

The datasets used and/or analyzed during the current study are available from the corresponding author on reasonable request.

\section{Authors' contributions}

DY and GL performed the research and drafted the manuscript. AL, FD and GC participated in the in vitro studies and performed the statistical analysis. WX established the rat model and performed the in vivo studies. YL and SH conceived the idea of the study, and participated in its design and coordination. All authors read and approved the final manuscript.

\section{Ethics approval and consent to participate}

The animal experiments were approved by the Animal Experimental Ethics Committee, The First Affiliated Hospital of Zhejiang University.

\section{Patient consent for publication}

Not applicable.

\section{Competing interests}

The authors declare that they have no competing interests.

\section{References}

1. Atkinson MA, Eisenbarth GS and Michels AW: Type 1 diabetes. Lancet 383: 69-82, 2014.

2. Chatterjee S, Khunti $\mathrm{K}$ and Davies MJ: Type 2 diabetes. Lancet 389: 2239-2251, 2017.

3. Gilbert RE and Krum H: Heart failure in diabetes: Effects of anti-hyperglycaemic drug therapy. Lancet 385: 2107-2117, 2015.

4. Katakami N: Mechanism of Development of atherosclerosis and cardiovascular disease in diabetes mellitus. $\mathbf{J}$ Atheroscler Thromb 25: 27-39, 2018

5. Low Wang CC, Hess $C N$, Hiatt WR and Goldfine AB: Clinical update: Cardiovascular disease in diabetes mellitus: Atherosclerotic cardiovascular disease and heart failure in type 2 diabetes mellitus-mechanisms, management, and clinical considerations. Circulation 133: 2459-2502, 2016.

6. Shi L, Ji Y, Jiang X, Zhou L, Xu Y, Li Y, Jiang W, Meng P and Liu X: Liraglutide attenuates high glucose-induced abnormal cell migration, proliferation, and apoptosis of vascular smooth muscle cells by activating the GLP-1 receptor, and inhibiting ERK1/2 and PI3K/Akt signaling pathways. Cardiovasc Diabetol 14: 18, 2015.

7. Carthew RW and Sontheimer EJ: Origins and mechanisms of miRNAs and siRNAs. Cell 136: 642e55, 2009.

8. He L and Hannon GJ: MicroRNAs: Small RNAs with a big role in gene regulation. Nat Rev Genet 5: 522-531, 2004.

9. Ding Y, Sun X and Shan PF: MicroRNAs and cardiovascular disease in diabetes mellitus. Biomed Res Int 2017: 4080364, 2017.

10. Rudijanto A: The role of vascular smooth muscle cells on the pathogenesis of atherosclerosis. Acta Med Indones 39: 86-93, 2007.

11. Chait A and Bornfeldt KE: Diabetes and atherosclerosis: Is there a role for hyperglycemia? J Lipid Res 50 (Suppl): S335-S339, 2009.

12. Chen GP, Zhang XQ, Wu T, Li L, Han J and Du CQ: Alteration of mevalonate pathway in proliferated vascular smooth muscle from diabetic mice: Possible role in high-glucose-induced atherogenic process. J Diabetes Res 2015: 379287, 2015.

13. Livak KJ and Schmittgen TD: Analysis of relative gene expression data using real-time quantitative PCR and the 2(-Delta Delta C(T)) method. Methods 25: 402-408, 2001.

14. Masiello P, Broca C, Gross R, Roye M, Manteghetti M, Hillaire-Buys D, Novelli M and Ribes G: Experimental NIDDM: Development of a new model in adult rats administered streptozotocin and nicotinamide. Diabetes 47: 224-229, 1998.

15. Matthews DR, Hosker JP, Rudenski AS, Naylor BA, Treacher DF and Turner RC: Homeostasis model assessment: Insulin resistance and beta-cell function from fasting plasma glucose and insulin concentrations in man. Diabetologia 28: 412-419,1985.

16. Hwang HW and Mendell JT: MicroRNAs in cell proliferation, cell death, and tumorigenesis. Br J Cancer 96: 776-780, 2007.

17. Ye D, Zhang T, Lou G and Liu Y: Role of miR-223 in the pathophysiology of liver diseases. Exp Mol Med 50: 128, 2018.

18. Maegdefessel L, Rayner KJ and Leeper NJ: MicroRNA regulation of vascular smooth muscle function and phenotype: Early career committee contribution. Arterioscler Thromb Vasc Biol 35: 2-6, 2015. 
19. Santovito D, Egea V and Weber C: Small but smart: MicroRNAs orchestrate atherosclerosis development and progression. Biochim Biophys Acta 1861 (12 Pt B): 2075-2086, 2016.

20. Ji R, Cheng Y, Yue J, Yang J, Liu X, Chen H, Dean DB and Zhang C: MicroRNA expression signature and antisensemediated depletion reveal an essential role of MicroRNA in vascular neointimal lesion formation. Circ Res 100: 1579-1588, 2007.

21. Liu X, Cheng Y, Zhang S, Lin Y, Yang J and Zhang C: A necessary role of miR-221 and miR-222 in vascular smooth muscle cell proliferation and neointimal hyperplasia. Circ Res 104: 476-487, 2009.

22. Leeper NJ, Raiesdana A, Kojima Y, Chun HJ, Azuma J, Maegdefessel L, Kundu RK, Quertermous T, Tsao PS and Spin JM: MicroRNA-26a is a novel regulator of vascular smooth muscle cell function. J Cell Physiol 226: 1035-1043, 2011.

23. Xu J, Li L, Yun HF and Han YS: MiR-138 promotes smooth muscle cells proliferation and migration in $\mathrm{db} / \mathrm{db}$ mice through down-regulation of SIRT1. Biochem Biophys Res Commun 463 1159-1164, 2015.

24. Cordes KR, Sheehy NT, White MP, Berry EC, Morton SU, Muth AN, Lee TH, Miano JM, Ivey KN and Srivastava D: miR-145 and miR-143 regulate smooth muscle cell fate and plasticity. Nature 460: 705-710, 2009.

25. Torella D, IaconettiC,Catalucci D, Ellison GM, Leone A, Waring CD, Bochicchio A, Vicinanza C, Aquila I, Curcio A, et al: MicroRNA-133 controls vascular smooth muscle cell phenotypic switch in vitro and vascular remodeling in vivo. Circ Res 109: 880-893, 2011.

26. Yang J, Chen L, Ding J, Fan Z, Li S, Wu H, Zhang J, Yang C, Wang H, Zeng P and Yang J: MicroRNA-24 inhibits high glucose-induced vascular smooth muscle cell proliferation and migration by targeting HMGB1. Gene 586: 268-273, 2016.

27. Chen M, Zhang Y, Li W and Yang J: MicroRNA-145 alleviates high glucose-induced proliferation and migration of vascular smooth muscle cells through targeting ROCK1. Biomed Pharmacother 99: 81-86, 2018.
28. Bi Q, Tang S, Xia L, Du R, Fan R, Gao L, Jin J, Liang S, Chen Z, $\mathrm{Xu} \mathrm{G}$, et al: Ectopic expression of MiR-125a inhibits the proliferation and metastasis of hepatocellular carcinoma by targeting MMP11 and VEGF. PLoS One 7: e40169, 2012.

29. Sun YM, Lin KY and Chen YQ: Diverse functions of miR-125 family in different cell contexts. J Hematol Oncol 6: 6, 2013.

30. Zhang Y, Zhang D, Lv J, Wang S and Zhang Q: MiR-125a-5p suppresses bladder cancer progression through targeting FUT4. Biomed Pharmacother 108: 1039-1047, 2018.

31. Cai M, Chen Q, Shen J, Lv C and Cai L: Epigenetic silenced miR-125a-5p could be self-activated through targeting Suv39H1 in gastric cancer. J Cell Mol Med 22: 4721-4731, 2018.

32. Yan L, Yu MC, Gao GL, Liang HW, Zhou XY, Zhu ZT, Zhang CY, Wang YB and Chen X: MiR-125a-5p functions as a tumour suppressor in breast cancer by downregulating BAP1. J Cell Biochem 119: 8773-8783, 2018.

33. Goldstein JL and Brown MS: Regulation of the mevalonate pathway. Nature 343: 425-430, 1990 .

34. Buhaescu I and Izzedine H: Mevalonate pathway: A review of clinical and therapeutical implications. Clin Biochem 40: 575-584, 2007.

35. Han J, Jiang DM, Du CQ and Hu SJ: Alteration of enzyme expressions in mevalonate pathway: Possible role for cardiovascular remodeling in spontaneously hypertensive rats. Circ J 75: 1409-1417, 2011.

36. Chen GP, Zhang XQ, Wu T, Han J and Ye D: Inhibition of farnesyl pyrophosphate synthase attenuates high glucose-induced vascular smooth muscle cells proliferation. Mol Med Rep 15: 3153-3160, 2017.

(i) $\Theta$ This work is licensed under a Creative Commons Attribution-NonCommercial-NoDerivatives 4.0 International (CC BY-NC-ND 4.0) License. 